\title{
Ethikkommissionen für physiotherapeutische Forschung - ein Diskurs
}

\section{Ethical Review Boards for Physiotherapeutic Research - A Discourse}

Autoren

Institute

\section{K. Reichel' ${ }^{1}$ K.-F. Heise ${ }^{2}$}

1 Institut für Gesundheits- und Pflegewissenschaft (IGPW), Charité - Universitätsmedizin Berlin

2 Research Center for Movement Control and Neuroplasticity, Group Biomedical Sciences, Katholieke Universiteit, B-Leuven

\section{Schlüsselwörter}

- Forschungsethik

- Ethikkommission

- gute wissenschaftliche Praxis

\section{Key words}

research ethics

- ethical review board

good scientific practice

\section{Bibliografie}

DOI http://dx.doi.org/ $10.1055 / \mathrm{s}-0035-1567143$ physioscience 2016; 12: 152-157 @ Georg Thieme Verlag KG Stuttgart · New York . ISSN 1860-3092

\section{Korrespondenzadresse Kathrin Reichel, M.Sc. \\ Charité - Universitätsmedizin Berlin \\ Institut für Gesundheits- und Pflegewissenschaft (IGPW) Augustenburger Platz 1 13353 Berlin \\ Kathrin.Reichel@charite.de}

\section{Dr. rer. nat.}

\section{Kirstin-Friederike Heise}

Katholieke Universiteit Leuven Research Center for Movement Control and Neuroplasticity Group Biomedical Sciences Tervuursevest 101 box 1501 3001 Leuven

Belgium

kirstin.heise@kuleuven.be

\section{Zusammenfassung}

\section{$\nabla$}

Ethische Fragen in der Forschung gewinnen auch international zunehmend an Bedeutung. In Deutschland bedarf es formal rechtlich noch immer keines offiziellen Ethikvotums zur Durchführung physiotherapeutischer Forschungsprojekte.

Dieser Beitrag ist ein eindeutiges Votum für die Anpassung an internationale Standards. Basierend auf der Entwicklung existierender Standards für Forschungsethik, liefert er zudem konkrete Vorschläge zur kurz- und mittelfristigen Umsetzung guter wissenschaftlicher Praxis für ethisch verantwortungsvolles Vorgehen in der Physiotherapieforschung unter Berücksichtigung der gegenwärtigen Situation in Deutschland.

\section{Einleitung}

"The protocol of the present study has been approved by the local ethical committee [...] number [...] and in accordance with the revised declaration of Helsinki [...]."

Welche Arbeitsschritte und organisatorischen $\mathrm{Zu}$ sammenhänge hinter diesem knappen Satz in einer Forschungsarbeit stecken, ist nicht unbedingt allen Lesern wissenschaftlicher Arbeiten klar; und noch viel weniger, welchen Fallstricken und Hürden insbesondere therapeutische Wissenschaftler beim Versuch, ein Ethikvotum in Deutschland zu erhalten, begegnen.

Die Schwierigkeiten beginnen schon lange, bevor es überhaupt um die Begutachtung therapeutischer Studienprotokolle geht. Oftmals ist nämlich gar nicht klar, welche Kommission für die vorhergehende Ethikberatung für eine solche Studie überhaupt zuständig ist. Einige zufällig ausgewählte Beispiele aus der jüngeren Vergangenheit zeigen, dass die Suche nach einer zuständigen Kommission in eine regelrechte Odyssee ausarten

\section{Abstract}

Ethical questions in research gain growing importance also internationally. In Germany there is still no formal legally need for an official vote of the ethical review board for the performance of physiotherapeutic research projects.

This article is a distinct vote for the adaptation to international standards. Based on the development of existing standards for research ethics, it also gives precise recommendations for the short-term and medium-term conversion of good scientific practice for ethically responsible physiotherapeutic research approaches considering the present situation in Germany.

und auch ohne Sirenen zu großer Verwirrung für die Wissenschaftler führen kann.

So musste eine Kollegin erfahren, dass sich die lokale medizinische Ethikkommission ihrer Alma Mater für eine „rein physiotherapeutisch ausgerichtete Studie“ nicht zuständig fühlte, obwohl ein Arzt an der Studie beteiligt war. Im selben Fall erfuhr die Wissenschaftlerin von der erst jüngst ins Leben gerufenen Ethikkommission des Deutschen Verband für Physiotherapie (ZVK), dass eben diese Studie nicht in den Zuständigkeitsbereich der ZVKEthikkommission falle, da ein Arzt beteiligt sei. In einem anderen Fall erhielt die forschende Kollegin von der Ethikkommission der lokalen Ärztekammer die Auskunft, dass eine physiotherapeutisch ausgerichtete diagnostische Studie keinerlei Ethikvotum benötige. Außerdem sei eine solche Studie ohnehin nicht durchführbar, da Physiotherapeuten gar nicht forschen dürften.

Diese nicht repräsentative Auswahl zeigt jedoch nicht nur, wie unübersichtlich sich die Lage für die wissenschaftlich tätigen Therapeuten gestaltet, sie ist auch im Ergebnis bitter, da eine spätere 
Veröffentlichung der geplanten Arbeit ohne Ethikvotum nahezu unmöglich ist. Auf internationaler Ebene ist die Publikation einer empirischen Arbeit in Fachzeitschriften mit Peer-review-Verfahren heute schon unmöglich, wenn ein Ethikvotum fehlt. Der Versuch, ein Abstract für die Konferenz der World Confederation for Physical Therapy (WCPT) einzureichen, führt ohne Ethikvotum zur Disqualifikation.

Aber braucht es denn überhaupt die Einschätzung einer unabhängigen Kommission, um ethisch verantwortungsvolle Forschung zu betreiben? Und was genau bedeutet es überhaupt, ethisch verantwortungsvoll wissenschaftlich zu arbeiten? Und nicht zuletzt: Wie kann ein Fachjournal wie die physioscience Wissenschaftler dabei unterstützen?

\section{Ethische Standards für physiotherapeutische Forschung in der Grauzone}

Anders als für Ärzte, deren Berufsordnung eindeutig festlegt, dass medizinische Forschungsvorhaben von einer Ethikkommission beraten und genehmigt werden müssen, besteht in Deutschland keine ebensolche rechtliche Verpflichtung für forschende Physiotherapeuten. Auch fachlich gesehen fehlt „[...] in Deutschland für Lehrende, Studierende und Forschende [...] ein Überblick sowie eine Handreichung dazu, welche ethischen Standards für Forschung in den therapeutischen Gesundheitsfachberufen hierzulande herangezogen werden können. Ebenso offen und zu diskutieren ist die Frage, ob oder in welchen Fällen eine Beratung durch eine Ethikkommission in Anspruch genommen werden sollte“ [12].

Warum in Deutschland Ethikkommissionen für Forschung am Menschen hauptsächlich an medizinischen Fakultäten und von Ärztekammern betrieben werden, ist in erster Linie historisch gewachsen. Dies mag mit der besonderen rechtlichen Stellung der ehemals sogenannten medizinischen Assistenzberufe in Deutschland zusammenhängen. Da geschichtlich begründet das therapeutische Handeln ausschließlich auf ärztliche Weisung stattgefunden hat, war auch eine eigenständige therapeutische Forschung unwahrscheinlich. Während die Emanzipation der therapeutischen Forschung in Deutschland langsam stattfindet, hat sich die Notwendigkeit international betrachtet immer aus bestehenden gesellschaftlichen Konstellationen und sozialpolitischen Situationen ergeben. Die Nachfrage nach ethisch verantwortungsvollem Handeln, wie z. B. die Beachtung von Datenschutz, ist jedoch insgesamt gestiegen und betrifft gleichermaßen alle Disziplinen wie die Psychologie und die Sozialwissenschaften.

\section{Entstehungsgeschichte und Hintergrund der Helsinki Deklaration \\ $\nabla$}

Die Frage, inwieweit Menschen zu Forschungszwecken herangezogen werden dürfen, stellte sich in der Medizin seit dem 17. Jahrhundert zunehmend durch eine Abkehr von der antiken Autoritätshörigkeit und einer Neuorientierung an moderner Naturwissenschaft und naturwissenschaftlichen Methoden einschließlich der Theorie des Experiments, der rational geplanten Empirie und der induktiven Methodik. Damit wurde der ausschließlich heilende Aspekt der Medizin um einen wissenschaftlichen erweitert, was zu einer Verschiebung der bis dahin geltenden ethischen Werte und der Notwendigkeit nach deren Ergänzung führte [5].

Infolge mehrerer revolutionärer Neuerungen in der Medizin, wie z.B. der Antisepsis, der Anästhesie und der wissenschaftlichen
Bakteriologie, kam es um 1900 nach den Experimenten mit Syphilis von Albert Neisser (1855 - 1916) zu weltweiten Diskussionen über die Verfahrensweise bei medizinischen Versuchen. Erstmals tauchte die Forderung nach der Zustimmung der Patienten auf. Von Gesetzgeberseite gab es erste Initiativen, Vorgaben bezüglich medizinischer Eingriffe zu Versuchszwecken zu erlassen, die forschungsethische Grundideen beinhalten wie den Schutz vulnerabler Gruppen, das Prinzip der informierten Zustimmung (Informed consent), die schriftliche Dokumentation sowie eine Klärung, wer forschen darf.

Der Verlauf der „Nürnberger Ärzteprozesse“ verdeutlichte dennoch, dass es zu dieser Zeit weder ein nationales noch internationales Dokument gab, welches sich mit der Legitimation von Humanexperimenten auseinandersetzte. Im Rahmen der Urteilsbegründung wurden die unter dem Namen „Nürnberger Kodex“ bekannten 10 Grundprinzipien zur Durchführung medizinischer Experimente am Menschen formuliert:

den körperlichen und geistigen Schutz der Versuchspersonen $(\S \S 4,5,6,7,9,10)$;

- die freiwillige, informierte Zustimmung der Versuchsperson $(\S 1)$;

die Abwägung von Nutzen und Risiko $(\S \S 2,5)$.

- Des Weiteren muss es dem Probanden (§9) und dem Versuchsleiter $(\S 10)$ freigestellt sein, den Versuch zu beenden. Als Abbruchskriterium werden wieder körperliche und geistige Schädigungen genannt.

- Mehrere Paragrafen regeln den Bereich, welcher heute unter die Wissenschaftlichkeit eines Versuchs subsumiert würde wie wissenschaftlich geschultes Personal ( $\S 8)$, Tierversuche im Vorfeld $(\S 3)$ und wissenschaftliche Planung des Versuchs ( $(3)$.

Forderungen nach einer Überarbeitung dieses stark rechtlich geprägten Dokumentes kamen auf, die unter anderem in der erstmals 1964 verabschiedeten Deklaration von Helsinki des Weltärztebundes (World Medical Association, WMA) mündete. Diese gibt Grundregeln für Studien mit Menschen, an identifizierbarem, d.h. auf eine Person zurückführbarem menschlichen Material oder mit identifizierbaren Daten vor. Zentrale Punkte sind die Freiwilligkeit der Teilnahme und Abbruchmöglichkeit, Aufklärung über Risiken und Nutzen bzw. Nichtnutzen, Datenschutz, Kompetenz der beteiligten Wissenschaftler sowie eine Unabhängigkeit von Sponsoren oder anderen äußeren Einflüssen. In $\vee \mathbf{A b b} \mathbf{1}$ ist die Deklaration in Überschriften zusammengefasst.

Die Deklaration richtete sich alleine aufgrund ihres Mandates des Weltärztebundes nur an Ärzte. Im Wissen um die an der Forschung am Menschen beteiligten Berufe ergänzt Art 2 in der aktuellen Fassung jedoch: „2. Im Einklang mit dem Mandat des WMA wendet sich die Deklaration in erster Linie an Ärzte. Der WMA regt andere an der medizinischen Forschung am Menschen Beteiligte an, diese Grundsätze zu übernehmen.“

Die Deklaration erhebt Anspruch auf weltweite Gültigkeit, ist im engeren Sinne jedoch kein international gültiges Recht und wird national auf unterschiedliche Weise in staatliches oder Berufsrecht umgesetzt. Sie stellt also eine mögliche Vorgabe für geltende Normen dar, ohne jedoch strenge Gültigkeit ins Recht fordern zu können [16]. Die 4 wesentlichen Aspekte Vulnerabilität der Studienteilnehmenden, Gewährleistung der informierten Zustimmung, Datenschutz bzw. Anonymisierung von Daten und ethische Prävention für Krisenfälle sind von zentraler forschungsethischer Bedeutung und finden sich sowohl in den Guidelines for Good Clinical Practice (GCP; von der WHO im Zusammenhang mit pharmakologischen Studien entwickelt $[10,11,15])$ als auch in der pflegewissenschaftlichen Forschung [13]. 
Zusammenfassung

Präambel

1. Geltungsbereich der Deklaration für medizinische Forschung am Menschen und an identifizierbaren menschlichen Materialien und Daten

2. Deklaration wendet sich in erster Linie an Ärzte, WMA regt andere an der medizinischen Forschung am Menschen Beteiligte an, diese Grundsätze zu übernehmen

Allgemeine Grundsätze

3.Gesundheit als oberstes Gebot ärztlichen Handelns, Handeln des Arztes ausschließlich im Interesse der Patienten

4. Pflicht des Arztes, die Gesundheit, das Wohlergehen und die Rechte der Patienten zu fördern und zu erhalten

5. Medizinischer Fortschritt muss sich auch auf Versuche am Menschen stützen

6. Ziel der medizinischen Forschung als Verbesserung prophylaktischer, diagnostischer und therapeutischer Verfahren und verbessertes Verständnis von Krankheitsentstehung und -verlauf

7. Medizinische Forschung unterliegt ethischen Standards, die die Achtung vor den Menschen fördern und sicherstellen und ihre Gesundheit und Rechte schützen

8. Vorrang des Wohlergehens der Versuchsperson vor den Interessen der Wissenschaft und der Gesellschaft

9. Pflicht des Arztes, die Versuchsteilnehmer zu schützen

10. Vorrangigkeit der Deklaration vor nationalen Gesetzen

11. Minimierung von Umweltschäden durch medizinische Forschung

12. Durchführung medizinischer Forschung am Menschen nur von dafür qualifizierten Personen

13. Zugang zur Forschungsteilnahme auch für in der medizinischen Forschung unterrepräsentierte Gruppen

14. Verbindung von Forschung und ärztlicher Behandlung

15. Angemessene Entschädigung und Behandlung für Versuchspersonen

Risiken, Belastungen und Nutzen

16. Ziel medizinischer Forschung am Menschen muss die Risiken und Belastungen für die Versuchspersonen überwiegen

17. Nutzen-Risiko-Abwägung vor jeder medizinischen Forschung am Menschen, Risikominimierung, -dokumentation und -überwachung

18. Beteiligung von Ärzten an Forschungsvorhaben unter der Bedingung, dass der potenzielle Nutzen überwiegt und Risiken beherrschbar sind

Vulnerable Gruppen und Einzelpersonen

19. Verpflichtung zu besonderem Schutz vulnerabler Gruppen und Einzelpersonen

20. Rechtfertigung medizinischer Forschung mit einer vulnerablen Gruppe nur, wenn sie nicht an einer nicht vulnerablen Gruppe durchgeführt werden kann und es der Gruppe nutzt

Wissenschaftliche Anforderungen und Forschungsprotokolle

21. Verpflichtung zu wissenschaftlichen Grundsätzen; Wissen aus Literatur und Labor- und Tierversuchen als Voraussetzung für medizinische Forschung, Schutz der Versuchstiere

22.Verpflichtung zur Führung eines Studienprotokolls mit der Rechtfertigung, Planung und Durchführung, ethischen Erwägungen; Informationen über Finanzierung, Sponsoren, institutionelle Verbindungen, mögliche Interessenkonflikte, Anreize für Versuchspersonen, ggf. Entschädigung usw. Forschungs-Ethikkommissionen

23. Verpflichtende Zustimmung durch eine Ethikkommission, Zustimmung bei Änderungen und Vorlage des Abschlussberichts

Privatsphäre und Vertraulichkeit

24. Vorsichtsmaßnahmen zum Schutz der Versuchspersonen und Vertraulichkeit persönlicher Informationen

Informierte Einwilligung

25.Voraussetzung der freiwilligen Zustimmung zur Teilnahme an Forschung

26. Aufklärung der Versuchspersonen bei der medizinischen Forschung

27. Informierte Einwilligung durch Dritte, wenn die Versuchsperson zum Arzt in einem Abhängigkeitsverhältnis steht

28.-30. Informierte Einwilligung nicht einwilligungsfähiger Personen

31. Information des Patienten über forschungsbezogene Aspekte

32. Einwilligung bei identifizierbaren menschlichen Materialien oder Daten

Verwendung von Placebos

33. Zulässigkeit der Verwendung von Placebo oder das Unterlassen einer Maßnahme

Maßnahmen nach Abschluss einer Studie

34. Für alle Teilnehmer, die noch eine Maßnahme benötigen, die in der Studie als nützlich erkannt wurde

Registrierung von Forschung sowie Publikation und Verbreitung von Ergebnissen

35. Verpflichtung zur Registrierung von Forschungsvorhaben mit Versuchspersonen vor der Rekrutierung in einer öffentlich zugänglichen Datenbank

36. Ethische Verpflichtung der Forscher, Verfasser, Sponsoren, Herausgeber und Verleger zur Veröffentlichung und Verbreitung der Forschungsergebnisse: Öffentliche Verfügbarkeit nach den Leitlinien für ethische Berichterstattung („Ethical reporting“) sowohl negativer als auch nicht schlüssiger

Ergebnisse, Darlegung von Interessenskonflikten, Finanzierungsquellen und institutioneller Verbindungen und Interessenkonflikten; Ablehnung von Forschungsberichten, die nicht mit den Grundsätzen dieser Deklaration übereinstimmen

Nicht nachgewiesene Maßnahmen in der klinischen Praxis

37. Heilversuche mit nicht nachgewiesenen Maßnahmen und Forschung

Grafik: K. Reichel; Umsetzung: Thieme Verlagsgruppe

Abb. 1 Zusammenfassung der Deklaration von Helsinki von 2013 [2]

\section{Zentrale forschungsethische Anforderungen: Vulnera- bilität, informierte Zustimmung und Datenschutz}

der oder an denen sie forschen. Sie befasst sich zudem mit den Maßnahmen, die zum Schutz der an der Forschung teilnehmenden Personen unternommen werden [13].
Wissenschaftsethik allgemein „reflektiert den moralischen Anspruch, unter dem die Wissenschaftler Wissenschaft betreiben“ [8], also z. B. wissenschaftliches Norm- und Fehlverhalten (z. B. Plagiarismus) im Allgemeinen, aber auch speziell die Durchführung von Forschung. Forschungsethik im engeren Sinne befasst sich mit der Frage, welche ethisch relevanten Einflüsse die Intervention von Forschern den Menschen zumuten könnte, mit

\section{Vulnerabilität}

Mit „Vulnerabilität“ ist allgemein die Verwundbarkeit bzw. Verletzbarkeit von Personen gemeint. Im Forschungskontext können dies Personen sein, die aufgrund ihrer eingeschränkten kognitiven oder physischen Fähigkeiten keine informierte Zustimmung geben und/oder wegen ihrer besonderen Lebenssituation durch die Teilnahme an einem Forschungsvorhaben in besonderem Maße belas- 
tet oder gar gefährdet werden könnten [13]. Daraus leitet sich eine besondere Schutzbedürftigkeit für den Umgang und die Teilnahmebedingungen an Forschungsvorhaben ab, was z. B. den Prozess der informierten Zustimmung oder auch den Umgang mit Gefährdung und Belastung durch die Teilnahme an Forschungsvorhaben betrifft. Zum Schutz nicht einwilligungsfähiger Personen in der medizinischen Forschung hat die Zentrale Ethikkommission bei der Bundesärztekammer eine Stellungnahme verfasst [18].

\section{Informierte Zustimmung}

Die informierte Zustimmung (Informed consent) meint die bewusste Zustimmung bzw. Einwilligung von Personen oder ihrer bevollmächtigten Vertreter, an einem Forschungsprojekt teilzunehmen und beinhaltet [13]:

- Ausreichende und verständliche Information;

- Ausreichend Zeit und Beratungsmöglichkeit, um sich eine Meinung bilden zu können;

- Keine Herbeiführung der Zustimmung durch Gewalt, Zwang, Betrug oder Täuschung;

- Für minderjährige oder bewusstlose Teilnehmende: Ersatz der persönlichen Einwilligung durch Zustimmung der gesetzlichen Vertreter und ebensolche Information und Zustimmungs- oder Ablehnungsmöglichkeit.

Die Studieninformation zur Erlangung der Einwilligung sollte demnach schriftlich in allgemein verständlicher Sprache verfasst sein und folgende Punkte umfassen [6]:

- Titel, Problemstellung und Ziele des geplanten Forschungsvorhabens und dessen Grenzen;

- Überblick über den derzeitigen Stand der Forschung;

- Darstellung des Studiendesigns und Ablauf des Forschungsvorhabens;

Darstellung der Nutzen-Risiko-Abwägung;

- Informationen zur Art der zu nutzenden Körpersubstanz, zum Zweck und Dauer der Verwendung;

Informationen zu Art und Ausmaß der Anonymisierung.

\section{Datenschutz}

Der Schutz und die Anonymisierung der erhobenen Daten der Forschungsteilnehmenden macht diese namenlos und schützt sie vor dem Zugriff der Öffentlichkeit. Unzureichender Datenschutz und Anonymisierung kann die Forschungsteilnehmenden gefährden, wenn diese z. B. in einem Abhängigkeitsverhältnis stehen, als Mitarbeiter oder Heimbewohner im Forschungsrahmen kritische Aussagen über die Versorgung treffen, die auf einzelne Personen zurückführbar sind.

Der Schutz personenbezogener Daten ist in Deutschland gesetzlich sowohl im Grundgesetz, als auch im Bundesdatenschutzgesetz (BDSG) und Strafgesetzbuch (StGB) geregelt. Die technische Umsetzung des Datenschutzes ist bei quantitativen statistischen Erhebungen meist unproblematisch, da die Daten häufig anonymisiert werden können, d.h. für die Forschung kein Personenbezug mehr notwendig ist. Zusätzlich besteht die Möglichkeit der faktischen Anonymisierung, d.h. der Personenbezug kann nur mit „unverhältnismäßig großem Aufwand“ hergestellt werden, die Pseudonymisierung von Daten ( $\S 3$ Abs. 6 BDSG). In erster Linie ist für den Datenschutz sicherzustellen, dass die Daten unzugänglich aufbewahrt werden, z. B. auf einem Rechner mit Passwortschutz, auf geschützt aufbewahrten mobilen Speichermedien oder als verschlossen abgelegte Dokumente. Forschungsdaten dürfen ausschließlich in anonymisierter Form weitergegeben werden. Zum Datenschutz in Forschungsprojekten gehört ebenso, die Rollen und Aufgaben der am Projekt Beteiligten festzuschreiben als auch ein lückenloses und organisiertes Berichts- und Dokumentationswesen zu führen, was auch die Dokumentation und Transparenz bei der Datenanalyse betrifft.

\section{Spannungsfelder in der Zusammenarbeit mit Ethikkommissionen}

Der grundsätzlich beratenden Rolle einer Ethikkommission und dem damit verbundenen Ziel, durch die Begutachtung und durch eventuelle Auflagen zur Überarbeitung des Projektplans die Qualität der Forschungsarbeit anzuheben, steht die Kritik gegenüber, Forschung durch ein bürokratisches Begutachtungsverfahren, Willkür und unnötige oder überzogene Auflagen eher zu behindern als zu unterstützen $[4,9,15]$. Gegner bemängeln, Ethikkommissionen hätten lediglich eine „Alibifunktion“ für den verantwortlichen Studienleiter $[4,9$, 15]. Hier wird unterstellt, dass die Forscher die Verantwortung für ihr Vorhaben damit an die Kommission delegieren und sich der Eigenverantwortung entziehen würden. Befürworter von Ethikkommissionen argumentieren, dass die sittliche und rechtliche Verantwortung immer bei den Forschenden selbst verbleibt. Ethikkommissionen seien vielmehr dazu geeignet, „das [...] Gewissen zu wecken, zu leiten und zu schärfen, aber auch es zu vergewissern und zu erleichtern" $[4,9,15]$.

Konkret lassen sich aus der Verfahrensperspektive hiesiger medizinischer Ethikkommissionen folgende Aspekte problematisieren und insbesondere für die therapeutischen Gesundheitsfachberufe kritisch prüfen, die bisher (noch) keinem weitergehenden und rechtsverbindlichen, ethischen Begutachtungsverfahren ihrer Forschungsvorhaben unterliegen:

Die Begutachtung durch eine Ethikkommission setzt einen Antrag der Forschenden voraus. Grundsätzlich beinhaltet dieses Vorgehen aber, dass die Forschungstätigkeit von den Initiatoren auch als solche anerkannt und deklariert wird. Die Verantwortung für eine ethische Beratung liegt also beim Initiator des Projektes. Dieser Aspekt scheint trivial, bedeutet jedoch in aller Konsequenz, dass, wenn eine Person ihre Tätigkeit nicht als Forschung bzw. Experiment, sondern eventuell als Entwicklungs- oder Evaluationsmaßnahme sieht, sie auch keinen Antrag auf ethische Prüfung stellen und das Vorhaben ohne Ethikvotum durchführen wird.

Darüber hinaus sind Ethikkommissionen in ihrer Beratungsfunktion auf eine zuverlässige Selbstauskunft der Antragstellenden angewiesen. Selten fungiert die Ethikkommission auch als Kontrollinstanz, und eine Prüfung der tatsächlichen Vorgehensweise ist nicht notwendigerweise vorgesehen. Dies leisten manche Ethikgremien, indem die Antragsteller im Sinne der guten wissenschaftlichen Praxis [3] vorab klare Kriterien für den Studienabbruch (z. B. beim Auftreten negativer Effekten im Zusammenhang oder als Folge der experimentellen Prozeduren) definieren und diese im Verlauf protokollieren und an die Ethikkommission melden müssen. Gleichermaßen erfragen manche Ethikkommissionen nach Abschluss des Projektes die Einhaltung des Projektplans.

Da sich die biomedizinische Forschung vorwiegend aus dem quantitativen Methodenkanon bedient, wird ein methodischer Bias kritisiert. So seien medizinische Ethikkommissionen eher am Vorgehen quantitativer Forschungsprojekte orientiert und würden deswegen qualitativ angelegter Forschung weniger gerecht [7, 9, 14]. Die gängigen Antragsformate für eine ethische Begutachtung sehen z. B. nicht vor, dass sich die Methode während der Durchführung entwickelt bzw. verändert, was qualitativen Forschungsmethoden immanent ist (zirkulärer Forschungsprozess). Das Problem wäre durchaus lösbar, z. B. durch Besetzung der Ethikkommission 
Tab. 1 Ethikkommissionen für physiotherapeutische Forschung in Deutschland

\begin{tabular}{|c|c|c|}
\hline Institution & Name der Kommission & Homepage \\
\hline \multicolumn{3}{|l|}{ Physiotherapeutische Berufsverbände } \\
\hline ZVK & $\begin{array}{l}\text { Ethikkommission des Deutschen Verbandes } \\
\text { für Physiotherapie (ZVK) e.V. }\end{array}$ & $\begin{array}{l}\text { www.physio-akademie.de/forschung-wissenschaft/ } \\
\text { wissenschaftliche-services/ethikkommission/ }\end{array}$ \\
\hline \multicolumn{3}{|c|}{ Hochschulen mit Studiengängen für Physiotherapie } \\
\hline $\begin{array}{l}\text { Alice Salomon Hochschule (ASH) } \\
\text { Berlin }\end{array}$ & Ethikkommission der ASH & www.ash-berlin.eu/forschung/ethikkommission \\
\hline $\begin{array}{l}\text { Hochschule für angewandte } \\
\text { Wissenschaft und Kunst (HAWK) } \\
\text { Hildesheim }\end{array}$ & Ethikkommission & www.hawk-hhg.de/hochschule/214 258.php \\
\hline Hochschule Fresenius Idstein & Ethikkommission & ethik.hs-fresenius.de \\
\hline $\begin{array}{l}\text { Hochschule für Gesundheit (hsg) } \\
\text { Bochum }\end{array}$ & $\begin{array}{l}\text { Die Ethikkommission der Medizinischen Fakultät der } \\
\text { Ruhr-Universität Bochum ist Partner der hsg Bochum } \\
\text { im Rahmen des Gesundheitscampus }\end{array}$ & www.ruhr-uni-bochum.de/ethik \\
\hline
\end{tabular}

mit entsprechender methodischer Expertise und Verfahrensänderungen, die ebensolchen Designs gerecht werden, wie internationale Beispiele zeigen.

Nicht nur im Hinblick auf die Forschungsmethodik, sondern auch in Bezug auf die spezifischen fachlichen Inhalte therapeutischer Forschung ist eine Berücksichtigung der fachspezifischen Expertise bei der Besetzung der Ethikkommission mehr als wünschenswert. Eine Öffnung der Ethikkommissionen an medizinischen Fakultäten für die forschungsethische Beratung von (physio-)therapeutisch initiierten Projekten wäre durchaus wünschenswert. Jedoch bleibt kritisch zu diskutieren, inwiefern diese Gremien vor dem Hintergrund ihrer Grundlagen und personellen Besetzung den Forschungsvorhaben aus den Gesundheitsfachberufen gerecht werden und verändert werden müssten.

\section{Möglichkeiten für therapeutische Wissenschaftler in Deutschland}

Konkret stellt sich anschließend an diese Ausführungen für forschende Therapeuten nun die Frage, wann bzw. in welchen Fällen es ratsam ist, für ein Forschungsprojekt eine Ethikkommission zur Beratung hinzuzuziehen. Letztendlich verbleibt die Verantwortung für die forschungsethische Durchführung eines Forschungsprojektes bei den Wissenschaftlern selbst; die Beratung durch eine Ethikkommission und „Genehmigung“ entbindet davon nicht, sollte aber unterstützende Funktion haben.

Welche Kommission für eine Ethikberatung infrage kommt, sollte im Einzelfall abgewogen werden, sowohl was die Erwartungen an die inhaltlich-fachliche Beratung als auch den finanziellen Aufwand und die zeitlichen Beratungsfristen betrifft. Alle Kommissionen arbeiten nach einer Satzung, die den Rahmen für ihre Tätigkeit vorgibt, d.h. die Mitglieder der Kommission, ihre Zuständigkeit bzw. wer Ethikanträge zur Begutachtung an die Kommission stellen kann und zu welchen Bedingungen (z. B. Gebühren, Fristen). Diese Gegebenheiten müssen unbedingt in den Forschungsprozess eingeplant werden. Neben den vorhandenen lokalen medizinischen Ethikkommissionen (Arbeitskreis Medizinischer Ethikkommissionen 2016; www.ak-med-ethik-komm. de) zeigt Tab. 1 Beispiele für Ethikkommissionen, die aufgrund ihrer Satzung für physiotherapeutische Forschungsvorhaben infrage kommen.

Unserer Meinung nach ist es unerlässlich, vor der Durchführung einer empirischen (physio-)therapeutischen Studie ein offizielles Ethikvotum einer entsprechenden Kommission einzuholen. Un- ter Abwägung der Möglichkeiten, eine Arbeit ohne das Votum eines externen Gremiums veröffentlichen zu können, sollte jedoch mindestens die kritische Auseinandersetzung mit den oben genannten Stichpunkten in schriftlicher Form transparent gemacht und dargelegt werden, und zwar:

1. Ethische Prognose und ethische Prävention: Vulnerabilitätsprüfung;

2. Gewährleistung der informierten Zustimmung;

3. Maßnahmen zum Datenschutz (z. B. Anonymisierung).

\section{Fazit und Quintessenz}

Auch wenn es (in Deutschland) formal rechtlich noch keines offiziellen Ethikvotums bedarf, votieren wir eindeutig dafür, nicht zuletzt um die (physio-)therapeutische Forschung in Deutschland weiterzuentwickeln und an internationale Forschungsstandards anzupassen. Nicht nur physiotherapeutische Forschung, sondern auch Wissenschaft entwickelt sich zunehmend in globalen Zusammenhängen, was nicht zuletzt einheitliche wissenschaftliche Standards verlangt [1].

Im Sinne der guten wissenschaftlichen Praxis erfordert die pragmatische Durchführung einer Studie in erster Linie, die Vulnerabilität der Studienteilnehmenden zu beachten, den Prozess der informierten Zustimmung angemessen zu gestalten, den Datenschutz zu beachten und sich bereits vor der Durchführung auf mögliche, kritische Szenarien vorzubereiten (ethische Prävention für Krisenfälle).

Nicht zuletzt kann eine wissenschaftliche Fachzeitschrift diesen Prozess fördern, indem sie von Autoren vor der Veröffentlichung ihrer Arbeiten die kritische Reflexion der oben genannten Aspekte und die Vorlage eines Ethikvotums verlangt. Um den Realitäten für physiotherapeutische Forschung (in Deutschland) Rechnung zu tragen und einen Übergang zu internationalen Standards zu fazilitieren, empfehlen wir kurz- und mittelfristig, dass Autoren in ihrem Beitrag zu den forschungsethisch kritischen Punkten im Text Stellung beziehen und spätestens im Rahmen des Peer-reviewVerfahrens in diesem reflexiven Prozess unterstützt werden.

\section{Literatur}

1 Bosch X. Safeguarding good scientific practice in Europe. EMBO Reports 2010; 11: 252-257

2 Bundesäztekammer. WMA Deklaration von Helsinki - Ethische Grundsätze für die medizinische Forschung am Menschen; 2013, www.bun- 
desaerztekammer.de/fileadmin/user_upload/downloads/pdf-Ordner/ International/Deklaration-von-Helsiniki_2013_DE.pdf (19.08.2016)

3 Deutsche Forschungsgemeinschaft (DFG). Sicherung guter wissenschaftlicher Praxis, in Sicherung Guter Wissenschaftlicher Praxis: Empfehlungen der Kommission "Selbstkontrolle in der Wissenschaft“. Weinheim: Wiley-VCH; 2013

4 Dixon-Woods M, Foy C, Hayden C et al. Can an ethics officer role reduce delays in research ethics approval? A mixed-method evaluation of an improvement project. BMJ Open 2016; 6: e011973

5 Haneder S. Die Helsinki Deklaration des Weltärztebundes - Forschungsethik und die Sozialisation der Medizinstudenten [Dissertation]. Freiburg: Albert- Ludwigs-Universität; 2008, www.google.de/search? q=vorgeschichtehelsinki+deklaration\&ie=utf-8\&oe $=u t f-8 \&$ client=firefoxb\&gfe_rd=cr\&ei=SKNGV_6cLIfZ8AfxnYHoBw\# (19.08.2016)

6 Klinkhammer G. Medizinische Ethikkommissionen: „Wirken ohne Reglementierung“. Dtsch Arztebl 2003; 100: A-3291/B-2739/C-2559

7 Oye C, Sorensen NO, Glasdam S. Qualitative research ethics on the spot: Not only on the desktop. Nursing Ethics 2016; 23: 455 - 464

8 Pieper A. Einführung in die Ethik. Tübingen: Francke; 2000

9 Pollock K. Procedure versus process: ethical paradigms and the conduct of qualitative research. BMC Medical Ethics 2012; 13: 25

10 Reichel K, Marotzki U, Schiller S. Ethische Standards für ergotherapeutische Forschung in Deutschland. Teil 1: Eine nationale und internationale Bestandsaufnahme. Ergoscience 2009; 4: 56-70

11 Reichel K, Marotzki U, Schiller S. Ethische Standards für ergotherapeutische Forschung in Deutschland. Teil 2: Mögliche Ebenen der Anwendung und Umsetzung. Ergoscience 2009; 4: 98-106
12 Reichel K. Zur Entwicklung von Ethikkommissionen für die therapeutischen Gesundheitsfachberufe - relevante Regularien, aktuelle Perspektiven und offene Fragen. In: Ringmann S, Siegmüller J (Hrsg) Ethische Aspekte in der Forschung mit Kindern. Perspektiven der Gesundheitsfachberufe. Frankfurt: Lang; 2013

13 Schnell MW, Heinritz C. Forschungsethik: Ein Grundlagen- und Arbeitsbuch für die Gesundheits- und Pflegewissenschaften. Bern: Huber; 2006

14 Seidel $M$, Mayer $H$. Die ethische Begutachtung von Pflegeforschung. Ausgewählte Ergebnisse einer empirischen Untersuchung. Pr-InterNet 2004; 12 : 687-690

15 Toellner $R$. Forschung und Folgenabschätzung - Von Nutzen und Nutzlosigkeit von Ethikkommissionen. In: Frewer A, Winau R (Hrsg). Grundkurs Ethik in der Medizin. Bd. 1: Geschichte und Theorie der Ethik in der Medizin. Erlangen: Palm \& Enke; 1997

16 Wiesing U, Ehni HJ. Die Deklaration von Helsinki des Weltärztebundes - Ethische Grundsätze für die Forschung am Menschen. In: Christian Lenk C, Duttge G, Fangerau H (Hrsg). Handbuch Ethik und Recht der Forschung am Menschen. Berlin: Springer; 2014

17 World Health Organization (WHO). Handbook for Good Clinical Research Practice (GCP); 2002, www.apps.who.int/prequal/info_gene$\mathrm{ral} /$ documents/gcp/gcp1.pdf (19.08.2016)

18 Zentrale Ethikkommission bei der Bundesärztekammer. Schutz nichteinwilligungsfähiger Personen in der medizinischen Forschung; 1997 www.zentrale-ethikkommission.de/page.asp?his=0.1.27 (19.08.2016) 